\title{
Prevalence and clinical presentation of lymphoproliferative disorder in patients with primary Sjögren's syndrome
}

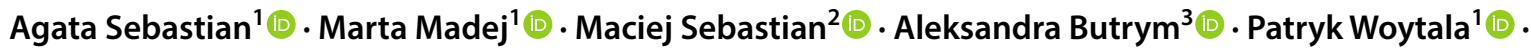 \\ Agnieszka Hałon $^{4}$ D . Piotr Wiland ${ }^{1}$ (D)
}

Received: 23 December 2019 / Accepted: 20 January 2020 / Published online: 1 February 2020

(c) The Author(s) 2020

\begin{abstract}
Lymphomas are one of the serious complications of the primary Sjörgen's Syndrome (pSS). The aim of the study was to evaluate the frequency of lymphoma in pSS. The singe-center retrospective study included 198 Caucasian patients, who met diagnostic criteria for pSS. The type of lymphoproliferative disorder was classified according to the WHO 2016 classification. The mean time of observation, after pSS diagnosis, was 48 weeks. Focus score (FS) $\geq 1$ was present in $85 \%$ of the patients, and anti-SSA antibodies were detected in $84 \%$. Rheumatoid factor was detected in $130(65 \%)$ patients. Mean disease activity index, according to EULAR Sjörgen's Syndrome disease activity index (ESSDAI), was 8.3 points at the moment of pSS diagnosis. Complement C 3 was decreased in $14 \%$ of the patients, while $10 \%$ showed reduced complement C4. Four patients (2\%) were diagnosed with a lymphoma. Most of the patients were diagnosed with mucosa-associated lymphoid tissue lymphoma (MALT), in whom the tumour was located in the parotid gland, and in one patient the stomach was involved. Finally, one patient was diagnosed with a rare B-cell small lymphocytic lymphoma located in the lungs. In this article, we present detailed characteristics of each case. In analysed population the frequency of lymphoma in the course of pSS in patients with pSS is $2 \%$. The variety of lymphoma types in pSS patients imposes individual monitoring in each patient at every check-up visit for disease activity.
\end{abstract}

Keywords Primary sjögren syndrome $\cdot$ Lymphoma $\cdot$ Monoclonal gammopathy

\section{Introduction}

One of the most severe complications of the primary Sjörgen's Syndrome (pSS) is lymphoma. pSS primarily involves exocrine glands, mainly the salivary and lacrimal glands, which causes the most characteristic symptom, i.e. dryness. However, lymphocytic infiltration may occur in practically every organ, which together with B-cell hyperactivation and

Agata Sebastian

agatasebastian@vp.pl

1 Department of Rheumatology and Internal Medicine, Medical University of Wroclaw, Borowska 213, 50-556 Wroclaw, Poland

2 Department of General, Minimally Invasive and Endocrine Surgery, Medical University of Wroclaw, Wroclaw, Poland

3 Department of Cancer Prevention and Therapy, Wroclaw Medical University, Wroclaw, Poland

4 Department of Pathomorphology and Oncological Cytology, Wroclaw Medical University, Wroclaw, Poland immunological disturbances may lead to developing lymphoma. Kassan et al. [1] were the first to describe the connection between development of lymphoma and SS in 1978. They showed that the risk of lymphoma in patients with SS is 44 times greater than in the general population. Today, the risk of lymphoma development in the course of pSS is, on average, 6-20 times greater than in the general population $[2,3]$. The most common lymphomas in the course of the pSS include the following: mucosa-associated lymphoid tissue lymphoma (MALT), nodal marginal zone lymphoma (NMZL) and diffuse large B-cell lymphoma (DLBCL) [4-6]. Generally accepted prediction factors of lymphoma associated with pSS include the following: reduced complement component $\mathrm{C} 4$, vasculitis-associated skin lesions and persistent or recurrent major salivary gland enlargement $[2,6]$.

The aim of this study was to evaluate the frequency of lymphoproliferative disorders in patients with pSS. 


\section{Patients and methods}

This retrospective study included 198 patients, who met the 2002 [7] and 2016 [8] diagnostic criteria for pSS, and were managed at the Rheumatology Department of the University Clinical Hospital. All of them were Caucasians. The mean age of the patients was 49 years. The type of lymphoproliferative disorder was classified according to the WHO 2016 classification-Classification of mature lymphoid, histiocytic and dendritic neoplasm [9]. The mean time of observation, after pSS diagnosis, was 48 weeks. The exclusion criteria included: age below 18 years, autoimmune comorbidities other than Hashimoto disease, e.g. secondary Sjögren syndrome. This retrospective study was approved by an appropriate local institutional review board (Medical University_Bioethics Committee number 357/2010 and 600/2018). All patients provided written informed consent to participate in the study.

\section{Statistical analysis}

To conduct analysis STATISTICA 10 software was used. For numerical variables descriptive statistics were calculated (mean and its $95 \%$ confidence interval, median, standard deviation, minimum and maximum values). For nominal variables frequency tables were calculated. These descriptive statistics were calculated for the group of patients not diagnosed with lymphoma (reference group). There were only four patients diagnosed with lymphoma and their medical parameters were compared with mean values of these parameters from the reference group.

\section{Results}

The studied group consisted of 191 (96\%) women and seven men (4\%). Dryness of the eyes was reported by $188(94 \%)$ of the patients, while xerostomia was reported by $182(91 \%)$ of the patients, those symptoms being compliant with the American-European Consensus Group (AECG) criteria [7]. Lymphocytic infiltration (Focus Score, FS), which is typical for pSS [10], was reported in 162 patients $(85 \%)$ of the patients, while anti-SSA antibodies were detected in $167(84 \%)$ patients. At the same time, seven $(3.5 \%)$ patients did not undergo baseline salivary gland biopsy. 29 (15\%) patients had negative FS. 27 of this patients did not have any pathological changes. Scattered lymphocytic infiltration was observed only in two patients, in an area of tissue more than $4 \mathrm{~mm}^{2}$. Rheumatoid factor (RF) was detected in $130(65 \%)$ patients. Mean activity of the disease, according to EULAR Sjörgen's syndrome disease activity index
(ESSDAI) [11], was 8.3 points at the moment of pSS diagnosis. Reduced complement component C3 was reported in $26(14 \%)$ patients, while decreased complement $\mathrm{C} 4$ was reported in $20(10 \%)$ patients. Detailed characteristics of the studied group is shown in Table 1.

Group of pSS patients with lymphoproliferative disorder (the characteristics of this group of patients is presented in Table 2).

In the studied group, four patients $(2 \%)$ including three women and one man were diagnosed with a lymphoproliferative disorder confirmed by specialist of histopathology, based on immunohistochemical investigations. Three patients $(75 \%)$ were diagnosed with MALT: in two of them it was located in the parotid gland and in one case the stomach was involved. One patient was diagnosed with a rare B-cell lymphoma, namely the small lymphocytic lymphoma, located in the lungs. In that patient (case number 1, Table 2), pSS was diagnosed in a woman younger than 55 and the first symptoms included a recurrent parotid gland enlargement as well as soft and hard palate tumour and dryness. On microscopic examination of the excised lesions (parotid glands, palate), the diagnosis of lymphoma, other neoplasm or IgG4-related disease were ruled out. A benign B-cell hyperplasia was diagnosed. Further tests objectively confirmed dryness, but also revealed diffuse dense infiltration of the labial salivary glands, typical for pSS, and a RF positive titer. The antinuclear antibodies (ANA) blood test result was negative. After 9 years, routine check-up high-resolution computed tomography (HRCT) of the chest showed peribronchial

Table 1 Characteristics of the studied group

\begin{tabular}{ll}
\hline & $\mathrm{NO} / \%$ \\
\hline pSS & 198 \\
Women & $191 / 96$ \\
Age of pSS diagnosis (year, medium) & $49(19-78)$ \\
Time of observation (month, medium) from pSS diag- & 48 \\
$\quad$ nosis & \\
Dry eye & $188 / 94$ \\
Dry mouth & $182 / 91$ \\
Focus score $<1$ & $29 / 15$ \\
LSGB not available & $7 / 3.5$ \\
Mean ESSDAI at pSS diagnosis & 8.3 \\
positive ANA $(\geq 1: 320)$ & $165 / 83$ \\
anty SSA antibodies & $167 / 84$ \\
positive RF $(>14$ IU/ml $)$ & $130 / 65$ \\
Low C3 complement $(<0.9 \mathrm{~g} / \mathrm{l})$ & $26 / 14$ \\
Low C4 complement $(<0.1 \mathrm{~g} / \mathrm{l})$ & $20 / 10$ \\
C3/C4 not available & 8 \\
\hline
\end{tabular}

$N O$ number, $p S S$ primary Sjögren syndrome, $L S G B$ lablial salivary gland biopsy, ESSDAI EULAR Sjörgen's syndrome disease activity index, $A N A$ antinuclear antibodies, $R F$ rheumatoid factor 
Table 2 Characteristics of pSS patients with lymphoma in the onset of lymphoproliferative disorder

\begin{tabular}{|c|c|c|c|c|}
\hline Patient numer & 1 & 2 & 3 & 4 \\
\hline Diagnosis & $\begin{array}{l}\text { B-cell lymphoma-small lympho- } \\
\text { cytic lymphoma (lungs) }\end{array}$ & $\begin{array}{l}\text { MALT (parotid } \\
\text { gland) }\end{array}$ & $\begin{array}{l}\text { MALT (parotid } \\
\text { gland) }\end{array}$ & MALT (stomach) \\
\hline $\mathrm{ESR}(\mathrm{mm} / \mathrm{hr})$ & 12 & 3 & NA & 20 \\
\hline Low C3 (nv 0.9-1.8 g/l) & 1.2 & 1.5 & NA & 1.3 \\
\hline Low C4 (nv 0.1-0.4 g/l) & 0.08 & 0.3 & NA & 0.2 \\
\hline RF (IU/ML, nv 0-14) & 78 & 5 & NA & 10 \\
\hline Lymphocyte (nv $1500-3500$ cells $/ \mathrm{ml}$ ) & 1600 & 870 & NA & 2000 \\
\hline $\operatorname{IgG}(\mathrm{nv}-7-16 \mathrm{~g} / \mathrm{l})$ & 15 & 8 & 16 & 14 \\
\hline $\operatorname{IgM}(\mathrm{nv} 0.4-2.3 \mathrm{~g} / \mathrm{l})$ & 2.1 & 2.0 & 0.6 & 0.8 \\
\hline $\operatorname{IgA}(\mathrm{nv} 0.7-4 \mathrm{~g} / \mathrm{l})$ & 2.1 & 2.0 & NA & 1.3 \\
\hline Palpable purpura & No & No & No & Yes \\
\hline Major salivary glands' enlargement & Yes & Yes & Yes & No \\
\hline ESSDAI-pSS diagnosis & 4 & 8 & 2 & 0 \\
\hline ESSDAI- lymphoma diagnosis & 19 & 8 & 8 & 9 \\
\hline Age in diagnosis pSS & 55 & 43 & 60 & 60 \\
\hline Age in diagnosis of limphoproliferative disease & 64 & 43 & 64 & 74 \\
\hline Focus score $\geq 1$ & Yes & No & Yes & No \\
\hline Anti-SSA antibodies & Negative & Positive & Negative & Positive \\
\hline Ann Arbor staging ${ }^{*}$ & I & I & I & I \\
\hline Treatment after pSS diagnosis & HCQ, GS & - & HCQ, GS & HCQ,GS \\
\hline
\end{tabular}

MALT extranodal marginal zone lymphoma of mucosa-associated lymphoid tissue, MGUS monoclonal gammopathy of undetermined significance, ESSDAI EULAR Sjörgen's syndrome disease activity index, $N A$ data not available, $R F$ rheumatoid factor; HCQ- hydroxychloroquine, $G S$ steroids

* Based on the reference number [12]

cuffing. Biopsy revealed benign B-cell hyperplasia. After 5 months, another HRCT scan was obtained due to the patient complaining on dyspnoea. The scan showed a tumour of the lung. The biopsy of the mass was performed and a preliminary diagnosis of lymphoma was made; however, because of the small size of the slice another biopsy was performed. B-cell small lymphocytic lymphoma was diagnosed. Despite chemotherapy the patient died within 12 months.

In the analysed group of pSS patients, the frequency of lymphoma was $2 \%$. In one case (case number 2, Table 2 ), the diagnosis of pSS was made synchronously with the diagnosis of salivary gland MALT, based on clinical symptoms (dryness) and positive anti-SSA antibodies. The mean ESSDAI score in a group with lymphoma, at the time of pSS diagnosis was 3.5 points on average and increased at the time of the last visit when lymphoma was recognized to 11 (not including lymph node/lymphoma domain). For the rest of the group without hyperplasia, mean ESSDAI score was 8.3 points at the moment of pSS diagnosis. In the subgroup of patients with lymphoproliferative disorders, palpable purpura was reported in one case and three of four patients in that subgroup presented major salivary gland enlargement. In one patient positive RF titer was noted. During the observation the level of immunologbulins was in normal range.

\section{Discussion}

According to the literature, the frequency of lymphoma in the population of patients with pSS is estimated to be between $2.7 \%$ and $9.8 \%$ in the Asian population [5]. For the European population, the precise frequency is unknown; however, it is known that the risk of lymphoma is about nine times higher than in the general population [4]. MALT is the most commonly diagnosed type of lymphoma in pSS patients [13]. One of the possible locations of MALT are lungs $[14,15]$. In described group of patients from our Department the frequency of lymphoproliferative disorders was $2 \%$ (mostly common being MALT).

As of today, there are no certain biomarkers of developing lymphoma in the course of pSS. In the recent years, there have been attempts to develop models which would increase the sensitivity of early lymphoma diagnosis in the population of pSS patients $[16,17]$. Some symptoms such as palpable purpura, salivary gland enlargement, low $\mathrm{C} 4$, leukopenia, positive cryoglobulins, monoclonal gammopathy, disease duration and positive RF are considered risk factors [18]. Applying such a broad model increases the sensitivity and specificity of more than $95 \%$ in identifying patients at risk of MALT [18]. Age, according to Chiu et al., also should be considered as an independent risk factor. Their research 
proved that patient's age correlates with the risk of monoclonal lymphocytic transformation estimated at $2.2 \%$ with each year of the patient's life [5]. However, as exemplified by our lymphoma cases, not all risk factors are always present. Only one patient showed positive RF titer, one patient had a reduced complement component, despite the fact that generally such abnormalities are observed in $65 \%$ and $10 \%$ of pSS patients, respectively. Thus, constant monitoring for lymphoma is necessary in pSS patients, including regular physical examination, laboratory tests and imaging.

It should also be mentioned that since 2016 lymphomas are no longer exclusion criteria of pSS, unlike the previous criteria [7, 8]. Diagnosis of pSS in a patient with lymphoma is acceptable as shown in our case of the man with salivary gland MALT. Nevertheless the diagnosis is sometimes difficult considering dryness, which may occur when lymphoma is located in the salivary gland and possible positive ANA in this population of patients. ANA are more frequently detected in patients with DLBCL before chemotherapy compared to the general population [19]. The speckled nuclear pattern is the most common. Presence of ANA may lead to development of a systemic connective tissue disease later on. Lang et al. showed that connective tissue disease was diagnosed in $10 \%$ of patients with DLBCL and positive ANA. Almost half of them developed SS, and specific anti-SSA/SSB antibodies were detected in those patients [19]. Based on the latest data from multicenter project the situation when patents were diagnosed with lymphoma and pSS synchronously is rare (1\% of patients) [20]. Patients diagnosed concomitantly with pSS and lymphoma have a very specific, highly active phenotype (men, white, severe oral involvement, cryoglobulinemic-related immunological markers, and high systemic activity) [20].

Based on the retrospective data from Swedish Register lymphoma was diagnosed in as much as $17 \%$ of the studied patients, either prior to pSS diagnosis or within 6 months since the diagnosis of pSS. Those patients were mostly men. Lymphadenopathy, MALT-type lymphoma and salivary gland lymphoma were also more common in these patients [21]. Similarly in our study, the patient with lymphoma diagnosed synchronously with pSS was male and suffered from MALT lymphoma. Less common neoplasms associated with pSS include oral and thyroid cancer [13], which have not been observed in our group.

Brito-Zeron et al. reported that the following risk factors of cancer development in pSS can be distinguished: high initial disease activity, presence of cryoglobulins accompanying hematological malignancies, cytopenia in the course of non B-cell lymphomas, decreased complement C3 associated with MALT or decreased component $\mathrm{C} 4$ and monoclonal gammopathy in the course of lymphomas other than MALT [22]. Monoclonal gammopathy of undetermined significance (MGUS) can potentially give rise to multiple myeloma. But still gammopathy itself is one of pSS symptoms [18]. MGUS can be referred to as a benign condition as there is only a small risk that MGUS can develop into myeloma or a related blood disorder. The average risk of progression to active myeloma is about $1 \%$ per year. Lifelong monitoring to detect any increase in the paraprotein level and development of symptoms is required. On the other hand, the risk of MGUS increases with age. About $3 \%$ of people over age 50 and $5 \%$ of people aged 70 and older have $\mathrm{M}$ protein in their blood. The highest incidence is among adults aged 85 and older. In our study MGUS was observed in $1.5 \%$ of patients (data not shown) and was not connected with lymphoma progression or other neoplasm. Elevated ESR was present in MGUS patients. However, ESRt was not consistently present in other lymphoproliferative disorders.

According to Baldini et al. [17], in women with pSS who later developed non-Hodkin lymphoma (NHL) the following features were present early in the course of the disease: salivary gland enlargement, skin purpura and/or peripheral nervous system involvement. The authors assumed the following as the risk factors of lymphoma: higher FS, decreased C4 level, positive cryoglobulins, and monoclonal gammopathy. It seems that FS and its intensity have a prognostic value in identifying patients at risk of abnormal lymphocyte proliferation. Risselda et al. reported that the FS was significantly higher in patients with NHL ( $3.0 \pm 0.894$ vs. $2.25 \pm 1.086$; $p=0.02$ ) [23]. FS $\geq 3$ had a positive predictive value for lymphoma development. Interestingly, the presence of Ig deposits (IgG, $\operatorname{Ig} \mathrm{A}, \operatorname{IgM})$ within the infiltrate showed no correlation with lymphoma development. Nevertheless, there was a correlation between $\mathrm{FS} \geq 3, \leq 40 \% \operatorname{IgA}+$ or $\geq 25 \% \mathrm{IgM}+$ and end-organ involvement. Recently, the possible clinical significance of MTHFR (methylenetetrahydrofolate reductase) gene polymorphism identification in the Caucasian race has been pointed out. More frequent occurrence of c. $667 \mathrm{C}>\mathrm{TT}$ genotype was reported in pSS patients who developed nonMALT lymphoma. No association between the presence of MTHFR gene and clinical symptoms has been noted, except for less frequent arthritis $(P=0.04)$ [24]. Also, there is current research being undertaken to identify single biomarkers of lymphoma such as CD30 cells. Ogawa et al. suggest that CD30 cells are increased in lacrimal glands and conjunctiva affected by SS and that a subset of SS patients are thereby at risk of developing lymphoma [25].

In the future, more precise determination would require larger study groups and comparison of medical registries from different geographical locations to determine the exact risk in local populations. The systemic phenotype of pSS is strongly influenced by indyvidual determinants such as age, gender, ethnicity and place of residence, which are key geoepidemiological players in driving the expression of systemic disease at diagnosis. The type of organ affected by pSS and the severity are modulated by geoepidemiological 
factors [26]. The risk factors for lymphoma are often unreliable and a variety of lymphoma types in pSS patients impose individual monitoring in each patient at every check-up visit for disease activity and possible lymphoma formation, not only at the moment of diagnosis. Our article is the first paper about the prevalence and types of lymphoproliferative disorders in East Central Europe.

Author contributions All authors contributed to the study conception and design, material preparation, data collection and analysis. All authors read and approved the final manuscript.

Funding This research received no specific grant from any funding agency in the public, commercial, or not for-profit sectors.

\section{Compliance with ethical standards}

Conflict of interest The authors declare that there is no conflict of interest.

Open Access This article is licensed under a Creative Commons Attribution 4.0 International License, which permits use, sharing, adaptation, distribution and reproduction in any medium or format, as long as you give appropriate credit to the original author(s) and the source, provide a link to the Creative Commons licence, and indicate if changes were made. The images or other third party material in this article are included in the article's Creative Commons licence, unless indicated otherwise in a credit line to the material. If material is not included in the article's Creative Commons licence and your intended use is not permitted by statutory regulation or exceeds the permitted use, you will need to obtain permission directly from the copyright holder. To view a copy of this licence, visit http://creativecommons.org/licenses/by/4.0/.

\section{References}

1. Kassan SS, Thomas TL, Moutsopoulos HM et al (1978) Increased risk of lymphoma in sicca syndrome. Ann Intern Med 89(6):888-892

2. Theander E, Vasaitis L, Baecklund E et al (2011) Lymphoid organisation in labial salivary gland biopsies is a possible predictor for the development of malignant lymphoma in primary Sjögren's syndrome. Ann Rheum Dis 70(8):1363-1368

3. Fox RI (2011) The importance of minor salivary gland biopsy in prediction of lymphoma in Sjögren's syndrome: should we be obtaining more information about prognosis from minor salivary gland samples? Ann Rheum Dis 70(8):1351-1353

4. Johnsen SJ, Brun JG, Gøransson LG et al (2013) Risk of nonHodgkin's lymphoma in primary Sjögren's syndrome: a population-based study. Arthritis Care Res (Hoboken) 65(5):816-821

5. Chiu YH, Chung CH, Lin KT et al (2017) Predictable biomarkers of developing lymphoma in patients with Sjögren syndrome: a nationwide population-based cohort study. Oncotarget 8(30):50098-50108

6. Voulgarelis M, Ziakas PD, Papageorgiou A (2012) Prognosis and outcome of non-Hodgkin lymphoma in primary Sjögren syndrome. Medicine (Baltimore) 91(1):1-9

7. Vitali C, Bombardieri S, Jonsson R et al (2002) Classification criteria for Sjogren's syndrome: a revised version of the European criteria proposed by the American-European Consensus Group. Ann Rheum Dis 61:554-558

8. Shiboski CH, Shiboski SC, Seror R et al (2017) 2016 American college of rheumatology/European league against rheumatism classification criteria for primary Sjögren's syndrome: a consensus and data-driven methodology involving three international patient cohorts. Ann Rheum Dis 76:9-16

9. Choi SM, O'Malley DP (2018) Diagnostically relevant updates to the 2017 WHO classificationof lymphoid neoplasms. Ann Diagn Pathol 37:67-74

10. Fisher BA, Jonsson R, Daniels T et al (2017) Standardisation of labial salivary gland histopathology in clinical trials in primary Sjögren's syndrome. Ann Rheum Dis 76(7):1161-1168

11. Seror R, Bootsma H, Saraux A et al (2016) Defining disease activity states and clinically meaningful improvement in primary Sjögren's syndrome with EULAR primary Sjögren's syndrome disease activity (ESSDAI) and patient-reported indexes (ESSPRI). Ann Rheum Dis 75(2):382-389

12. Cheson BD, Fisher RI, Barrington SF et al (2014) Recommendations for initial evaluation, staging, and response assessment of Hodgkin and non-Hodgkin lymphoma: the Lugano classification. J Clin Oncol 32:3059-3067

13. Brito-Zerón P, Kostov B, Fraile G et al (2017) Characterization and risk estimate of cancer in patients with primary Sjögren syndrome. J Hematol Oncol 10(1):90

14. Yachoui R, Leon C, Sitwala K, Kreidy M (2017) Pulmonary MALT lymphoma in patients with Sjögren's syndrome. Clin Med Res 15(1-2):6-12

15. De Vita S, Gandolfo S (2019) Predicting lymphoma development in patients with Sjögren's syndrome. Expert Rev Clin Immunol 15(9):929-938

16. Retamozo S, Brito-Zerón P, Ramos-Casals M (2019) Prognostic markers of lymphoma development in primary Sjögren syndrome. Lupus 28(8):923-936

17. Baldini C, Ferro F, Luciano N, Bombardieri S, Grossi E (2018) Artificial neural networks help to identify disease subsets and to predict lymphoma in primary Sjögren's syndrome. Clin Exp Rheumatol 112(3):137-144

18. Lang J, Ma K, Guo J, Zhang J, Wang Q, Sun H (2018) Clinical significance of elevated antinuclear antibodies in patients with diffuse large B-cell lymphoma: a single center study. J Cancer Res Ther 14(1):213-219

19. Fang YF, Chen YF, Chung TT et al (2017) Hydroxychloroquine and risk of cancer in patients with primary Sjögren syndrome: propensity score matched landmark analysis. Oncotarget 8(46):80461-80471

20. Soledad R, Nihan AD, Wan F et al (2019) OP0126 Lymphoma arising at the time of diagnosis of primary Sjögren syndrome: a highly-active systemic subset of the disease. In: Annual European congress of rheumatology, EULAR 2019, Madrid, 12-15 June 2019. https://doi.org/10.1136/annrheumdis-2019-eular.2591

21. Vasaitis L, Nordmark G, Theander E et al (2018) Comparison of patients with and without preexisting lymphoma at diagnosis of primary Sjögren's syndrome. Scand J Rheumatol 48(3):1-6

22. Brito-Zerón P, Retamozo S, Gandía M et al (2012) Monoclonal gammopathy related to Sjögren syndrome: a key marker of disease prognosis and outcomes. J Autoimmun 39(1-2):43-48

23. Risselada AP, Kruize AA, Goldschmeding R et al (2014) The prognostic value of routinely performed minor salivary gland assessments in primary Sjögren's syndrome. Ann Rheum Dis 73(8):1537-1540

24. Fragkioudaki S, Nezos A, Souliotis VL et al (2017) MTHFR gene variants and non-MALT lymphoma development in primary Sjogren's syndrome. Sci Rep 7(1):7354

25. Ogawa A, Ogawa Y, Mukai S et al (2019) Cluster of differentiation 30 expression in lacrimal gland and conjunctival tissues in 
patients with Sjögren's syndrome: case series. Medicine (Baltimore) 98(29):e16390

26. Brito-Zerón P, Acar-Denizli N, Ng WF et al (2019) Epidemiological profile and north-south gradient driving baseline systemic involvement of primary Sjögren's syndrome. Rheumatology (Oxford). https://doi.org/10.1093/rheumatology/kez578
Publisher's Note Springer Nature remains neutral with regard to jurisdictional claims in published maps and institutional affiliations. 